\title{
Double Negation and Negative Concord in English Language
}

\author{
Pangkuh Ajisoko $^{1 *}$, Arfha Rizky Firdausya ${ }^{2}$, Sherly Natalina ${ }^{3}$, Khaerawaty \\ Darwis $^{4}$
}

\author{
1, 2, 3,4English Education Department, Borneo University of Tarakan, Tarakan, Indonesia \\ Corresponding Author*Email: Pangkuh.ajisoko@gmail.com
}

\begin{abstract}
Double negation and negative concord statements content negative markers and express exceptions or unusual occurrences. The purpose of this article is to investigate the process of interpretation for hearers to be able to interpret sentences into either negative concord or double negation sentences. A literature review was used as the method of this article. Two experiments were reviewed in this article. It is implied that the preference of negative concord or double negative interpretations is a bias toward which negation style is acquired first during the linguistic developmental phase of children. It is also implied that redundant information, such as negative concord sentences, is more easily processed than more complex sentences such as double negation sentences. However, evidence state that standard English permits no use of negative concord.
\end{abstract}

Keywords: Double negation, negative concord, interpretation

\section{INTRODUCTION}

Humans generally communicate in positive terms and use negation to express exceptions or unusual occurrences. Negation statements contain negative markers, including not, n't, or never. In philosophy and linguistics, negotiation is generally accepted as conveying positive meaning [1].

In languages, two negative markers can cancel each other out and, in turn, creates a nonnegated form of words; thus, logically, two negative markers result in a positive one. For example, not impossible is logically equivalent to possible [2]. However, if a negative word is attached to the same word, usually when a word is not placed before a word containing a negative prefix, the expression's result is somewhat different from the idea mentioned earlier that two negative elements produce a positive one. For example, not without a doubt does not precisely mean with some doubt, and not uncommon has a weaker meaning than common [3]. This phenomenon is also known as double negation. Double negation, used to create meiosis or understatement, is commonly cited as one of the usual ways of indirectly rendering meaning in language [4].

Double negation interpretations exist alongside negative concord interpretations. In negative concord, sentences with two negation elements may be interpreted as sentences with a single negation [5]. For example, the sentence Maria didn't eat nothing today would be interpreted as Maria ate nothing today, whereas, in double negation, the same sentence would be construed as Maria ate something today.

In negative concord, two negative markers act in concord, resulting in one single semantic negation. In contrast, in double negation, two negative markers work independently to the semantics and form a double negation form of the sentence, equivalent to an affirmative (positive) sentence [6]. Negative concord is generally regarded as non-standard English. In contrast, double negation is usually considered standard English. However, some theoretical literature has raised the possibility that standard English might be a negative concord language [7]. A sentence with two negation markers combined with a particular set of negative expressions, including nobody; nothing; nowhere. It May have both negative concord and double negation interpretations in that sentence alone, thus, may be ambiguous [5]. Therefore, the need to use double negation and negative concord in writing and speaking is worth investigating. 


\section{METHODS}

This article uses a literature study as its research method. It uses a perception task based on the production of sentences with two negatives by standard English speakers.

In the study carried out by [5], twenty children and fifteen adults, as participants, were assigned to interpret sentences that were ambiguous between negative concord and double negation. This experiment hypothesized that children tend to assign negative concord interpretations, whereas adults tend to assign double negation interpretations. The null hypothesis of this experiment was that English is a double negation for both children and adults. The test sentences contained two negative markers, sentential negation followed by nothing; the example is as follows:

Test sentence: The boy who skipped didn't purchase nothing.

1. The boy who skipped purchased something (DN)

2. The boy who skipped purchased nothing (NC)

Results showed that 15 out of 20 children preferred negative concord interpretations, three children preferred double negation interpretations, and two children had no preference. Questions with negative quantifiers nothing provided evidence that standard English is a double negation language. In contrast, thirteen adults preferred double negation interpretations, and two adults had no preference.

\section{DISCUSSION}

Standard and non-standard English are varieties or dialects spoken related to the intellectual, social, and cultural point of view [8]. English is known to be a standard language, although there are studies that argue this and state that English might as well be a non-standard language. In standard English, each negative marker in a sentence contributes an independent semantic negation, and two negative elements cancel each other out, resulting in an affirmative interpretation. However, due to its complexity, double negation is thought to have a more significant processing cost than negative concord or single negation [9]. In contrast, negative concord is considered to be nonstandard English. In a negative concord, two negative markers act in concord and form one semantic negation [6].

It is understood that there exist double negation and negative concord languages. Languages are learned as children develop and grow. According to [5], after a child learns what kind of language they are getting, their linguistic performance will be biased towards the language learned, either double negation or negative concord. This biased behavior is predicted to solidify as the child continues to grow and develop.

According to Coles-White [10], the study showed that negative concord is preferred to interpret potentially ambiguous sentences. It is due to the difficulty to compute double negation interpretations as it is inherently problematic. This study is in sync with the research carried out by Thornton [5]. Thornton supposes that children have acquired negation forms, such as $-n$ 't, once they have begun to produce sentences with the negative auxiliary verb doesn't. Therefore, it is assumed that the participants of Thornton's experiment hypothesize that English permits negative concord. However, there is evidence that standard English does not allow negative concord. The children possible to suffer from more significant limitations in processing resources and theories. Compared to adults and therefore interpreted sentences in negative concord, there is evidence that standard English does not allow negative concord. In this experiment, the children were also considered not as sophisticated as pragmatics as adults in the same test. The latter mainly interpreted double negation sentences as double negation interpretations. Thus, once they develop their sense of pragmatism, they, too, would resort to using double negation interpretations accordingly. According to [9], it was assumed that single negation sentences were unproblematic for English speakers. Therefore double negation sentences might be unacceptable. The same study conducted by [9] also states that participants who are exposed. Adopting negative concord language read double negation sentences much more slowly and with more errors than reading negative concord sentences.

Coles-White also speculates that redundant information, such as negative concord, is more easily processed than additional negative information, such as double negation. In [11], they also states that it is assumed that interpreting an ambiguous sentence to make the sentence correct is more natural than to make the sentence false. This phenomenon is called The Principle of Charity. When hearers are confronted with an ambiguous sentence, they tend to make an effort to interpret the sentence to make it comport with reality. This is also called a "Yes" bias.

Deprez's [12] proposes that there might be evidence for negative concord in double negation languages, such as standard English. However, this argument is complicated because the language of negative concord is very stigmatized socially in an English-speaking society. It is because theoretically, in English, two 
negatives must be equal to positive. Negative concord, interestingly, appears to own an ability to distinguish standard from non-standard English speakers. A study by [13] illustrates that negative concord language is of vernacular English and that the shift away from using negative concord English was a marker of climbing social status.

Standard English is perceived as having more status and more prestigious than any other English dialect due to its association with professional societies. On the other hand, nonstandard English is considered blue-collar English, which is used casually in the neighborhood [14]. Non-standard English might use original negation features, including ain't, innit, and never [15]. However, Blanchette (2013) proposed that despite some people's unacceptability toward non-standard English, it is generated by all English varieties, which also include standard English. Thus, speakers who reject the use of non-standard English should at least own grammatical knowledge of it.

Aside from usually logically creating an affirmative, double negation has the pragmatic function of contradicting or correcting a previous negative statement. The double negation, as the standard English used in social life, is used to make meiosis or underestimate. It is intended to reduce or increase the intensity of the nature of actions, objects, activities, etc. The term meiosis, however, is most often used to denote negative assertion. This phenomenon also goes by the term litotes (for example, It is not unreasonable). Meiosis can lessen the candor of a statement, which sometimes can even be considered as goodwill. For instance, It would not be unfair to say she did not look her best.

In contrast, double negation can also be used to increase the intensity of a statement; for example, the expressions not unselfish, not inexpensive, and not undemanding are used ironically to mean highly selfish, highly expensive, and highly demanding. Thus, different double negatives contribute essential nuances to the different contexts of utterance [4]. This study also implicated that double negative enables us to interpret the meaning of utterances that are not specifically affirmative or negative.

Syntactic structure plays a role in the preference toward either double negation or negative concord. Grammar, in general, can generate double negation and negative concord. The study shows that the existence of the $n$ ' $t$ marker relative to negative phrases influences the interpretation of the sentences, in which negative concord is preferred when the tag precedes the negative expression (e.g., He didn't take nobody on the trip). Double negation is chosen when the marker follows the negative phrase (e.g., Nobody didn't help patients on that day) [16]. This study highlighted the importance of negative markers' relativity to negative subjects in shaping either interpretation. These results are synchronized with the generalization of Zeijlstra's typology [17]. If a language has a negative marker that is the syntactic head, then the final product is a negative concord language. Thus, if the negative marker is not analyzed as ahead in English, standard English has predicted it is a negative concord language. It is contrary to conventional assumptions that regard standard English as a double negation language.

A study carried out by [6] also provides evidence that, along with the syntactic structure, the speaker's intent plays a role in shaping interpretation. However, its position is not as privileged as the $-n$ 't marker itself. Acoustic cues can determine the production of either negative concord or double negation. It is assumed that negative concord speakers would use prosodic cues reliably, while dual negative speakers would not. Blanchette's study showed that the speaker tended to bias toward object negative concord interpretations and subject double negation interpretations when the sentences included the marker $-n$ 't. Double negation interpretation was marked relative to negative concord and single negation in the object condition. In which a shorter duration on stressed syllables, such as nothing and won't, were present. Thus, double negation is marked relative to negative concord prosodically. According to [5], double negation sentences are often associated with phonological stress on the second marker. Speakers may tend to default to negative concord interpretations in the absence of these phonological stress.

It is thought that standard English speakers do not use negative concord interpretations. However, studies reviewed above stated that grammars might have latent structures. Such structures can be generated but are not necessarily used (such as negative concord). Those are used for nonlinguistic reasons such as normative pressure and the evidence that ungrammatical can be interpreted reliably. In negative concord English, it can be seen that the normative force defines its use and acceptability.

It is known that the acceptability of usage of double negation and negative concord relies on syntactic preferences and acoustic cues to interpret the audio stimuli. Thus, it can be concluded that both negative concord and double negation as both grammatical in English. 


\section{CONCLUSION}

Double negation is a phenomenon in which two negative markers cancel each other out and produce a positive meaning. Negative concord is a phenomenon in which two negative tags act in harmony to create sentences with a single negation. Double negation language is considered standard English, and it exists alongside negative concord language, which is deemed non-standard English. Double negation language is considered to be more prestigious and sophisticated compared to negative concord. However, English speakers tend to understand the concept of negative concord despite rejecting negative concord use. Negative concord is typically used as a form of everyday language. Aside from prestige, interpreting sentences into either double negation or negative concord interpretation is a bias toward which language is acquired first during the linguistic developmental phase of children. Standard English speakers rely on syntactic information and acoustic cues in speech signal to choose between negative concord and double negation interpretations. Speakers show a tendency to select either interpretation that makes the sentence correct. Double negation interpretations can also be used to lessen or higher the property of adjectives or verbs in a sentence.

\section{REFERENCES}

[1] Z. Sarabi and E. Blanco, "Understanding negation in positive terms using syntactic dependencies," EMNLP 2016 - Conf. Empir. Methods Nat. Lang. Process. Proc., no. 3, pp. 1108-1118, 2016, doi: $10.18653 / \mathrm{v} 1 / \mathrm{d} 16-1119$.

[2] N. O. Schiller et al., "Solving the problem of double negation is not impossible: electrophysiological evidence for the cohesive function of sentential negation," vol. 3798, 2017, doi: 10.1080/23273798.2016.1236977.

[3] S. Smith, "Relations of Inclusion," Language (Baltim)., vol. 48, no. 2, pp. 276-284, 1972, doi: 10.2307/412135.

[4] M. M. Philippova, "Double and Multiple Negation in English,” pp. 1-8, 2003.

[5] R. Thornton, A. Notley, V. Moscati, and S. Crain, "Two Negations for the Price of One,” Glossa a J. Gen. Linguist., vol. 1, no. 1, pp. 1-30, 2016, doi: 10.5334/gjgl.4.

[6] F. Blanchette, M. Nadeu, J. Yeaton, and V. Deprez, "English negative concord and double negation: The division of labor between syntax and pragmatics," Linguist. Soc. Am., vol. 3, no. 53, pp. 115, 2018, doi: https://doi.org/10.3765/plsa.v3i1.4349.

[7] R. Zwaan et al., Sentential Negation and Negative Concord, vol. 29, no. 2. 2006.

[8] P. Trudgill, "Sociolinguistics: An introduction to language and society," Penguin Books, p. 243, 2000.

[9] I. de-Dios-flores, "Processing sentences with multiple negations: Grammatical structures that are perceived as unacceptable," Front. Psychol., vol. 10, no. OCT, 2019, doi: 10.3389/fpsyg.2019.02346.

[10] D. Coles-White, "Negative Concord in Child African American English: Implications for Specific Language Impairment," J. Speech, Lang. Hear. Res., vol. 47, no. 1, pp. 212-222, 2004, doi: 10.1044/1092-4388(2004/018).

[11] J. Cubillos, "Donald Davidson, Inquiries into Truth and Interpretation 2001 (Second Edition) ," no. December 1966, pp. 1-145, 2004, [Online]. Available: papers3://publication/uuid/D80362A95906-4925-BF32-372277807C71.

[12] V. Déprez, Atoms of negation: An outside-in micro-parametric approach to negative concord, no. January 2012. 2012.

[13] T. Nevalainen, "Social Mobility and the Decline of Multiple Negation in Early Modern English," in Advances in English Historical Linguistics, J. Fisiak and M. Krygier, Eds. Berlin: Mouton de Gruyter, 1996, pp. 263-291.

[14] H. P. Guth, Guth, Hans P English for a New Generation.USA: McGraw-Hill, Inc. USA: McGraw-Hill, Inc, 1973.

[15] I. P. Martínez, "Non-Standard Negation in Modern English: A Corpus-Based Study of Four Salient Features," ES Rev. Filol. Inglesa, vol. 34, no. 08, pp. 211226, 2013, [Online]. Available: https://core.ac.uk/download/pdf/2111009 82.pdf.

[16] F. Blanchette, "Micro-syntactic variation in American English Negative Concord," Glossa a J. Gen. Linguist., vol. 2, no. 1, p. 65, 2017, doi: 10.5334/gjgl.188.

[17] H. Zeijlstra, "Negative Concord is Syntactic Agreement," Ms., Univ. Amsterdam, vol. 5, no. 4, pp. 1-53, 2008. 